\title{
LE VERSIONI ITALIANE DELLA STORIA DI FIORAVANTE
}

\author{
NICOLETTA ILARIA BARBIERI (*) \\ Nota presentata dal s.c. Giuseppe Frasso \\ (Adunanza del 26 maggio 2011)
}

SunTO. - Dopo il ritrovamento di G. Contini del codice Conv. Soppr. da Ord. Badia 30 della BNCF, non è stato ancora condotto alcuno studio sistematico sulla Storia di Fioravante in esso contenuta. Questo contributo si propone di dimostrare, mediante confronti testuali, che questo romanzo cavalleresco è una redazione autonoma della vicenda di Fioravante attestata in altre quattro compilazioni italiane.

$$
* * *
$$

Aвstract. - After G. Contini's finding of the codex Conv. Soppr. da Ord. Badia 30, stored in BNCF, no systematic study has been carried out about the Storia di Fioravante contained in the codex. This paper aims at demonstrating, through textual comparisons, that this chivalric romance is an autonomous version of Fioravante's tale documented in other four Italian compilations.

Quando Douglas Mc Arthur pubblicò il suo contributo sulle redazioni del Libro di Fioravante, aveva il proposito esplicito di offrire un elenco, accompagnato da una sommaria descrizione, di tutte le compilazioni prodotte in area italiana che, a suo parere, corrispondevano

(*) Università Cattolica del Sacro Cuore, Milano, Dipartimento di Studi medievali, umanistici e rinascimentali. E-mail: nicolettailaria.barbieri@unicatt.it 
alla Chanson de Floovant tràdita dal ms. 441 della Bibliothèque de la Faculté de Médecine di Montpellier. ${ }^{1}$

L'analisi comparativa preliminare effettuata dal filologo metteva in luce che tali compilazioni, pur essendo accomunate dal fatto di essere incentrate sulle avventure guerresche e sentimentali del cavaliere Fioravante, manifestano ciascuna specificità proprie e sono a vario titolo in rapporto le une con le altre.

Le redazioni vagliate da Mc Arthur sono quattro: Il Libro delle Storie di Fioravante, I Reali di Francia di Andrea da Barberino, il Libro di Fioravante e Il Romanzo di Francia. ${ }^{2}$ Tutti questi romanzi cavallereschi in prosa, che si distinguono per la forma e per il contenuto, non-

1 Mc Arthur D. Les versions du "Libro di Fioravante". Filologia romanza 7, 1960, pp. 121-128: 120-121. Sul testo della Chanson de Floovant conservata a Montpellier, si vedano lo studio Darmesteter A. De Floovante vetustiore Gallico poemate et de Merovingico cyclo scripsit et adjecit primum Olavianam Flovents sagae versionem et excerpta e Parisiensi codice "Il Libro de Fioravante"..., Vieweg, Lutetiae Parisiorum, 1877 e le seguenti tre edizioni dell'opera con relative prefazioni: Michelant H. Guessard F. Gui de Bourgogne, Otinel, Floovant, in Les anciens poètes de la France, I, Jannet, Paris, 1858; Bateson H. La chanson de Floovent, étude critique et édition, Loughborough, Paris, 1938; Andolf S. Floovant, chanson de geste du XII siècle, Almqvist\&Wiksells, Uppsale, 1941. Delle compilazioni segnalate da Mc Arthur D., in particolare del Libro di Fioravante, mi sono occupata nella mia tesi di laurea magistrale (discussa presso l'UCSC di Brescia nell'a.a. 2006/2007, relatore prof. Andrea Canova) Il "Libro di Fioravante" (Parigi, Bibliothèque Nationale, ms. it. 1647): origini, edizione e commento e nell'articolo Un romanzo cavalleresco copiato a Pontevico nel Quattrocento, in Il libro fra autore e lettore, a c. di Grohovaz V., Atti della terza giornata di studi "Libri e lettori a Brescia tra Medioevo ed età moderna", 21 novembre 2006, La Compagnia della Stampa Massetti Rodella Editori, Brescia, 2008, pp. 25-39. Esprimo il mio più sincero ringraziamento ai professori Andrea Canova, Giuseppe Frasso e Paolo Gresti per avermi guidata nella realizzazione di tali lavori. L'edizione del Libro di Fioravante è attualmente in corso di preparazione.

2 Si tratta di quattro romanzi cavallereschi in prosa, dei quali i primi due sono stati pubblicati, rispettivamente, in Rajna P. Ricerche intorno ai "Reali di Francia" seguite dal "Libro delle Storie di Fioravante" e dal "Cantare di Bovo d'Antona", I, in I Reali di Francia, Romagnoli, Bologna, 1872, pp. 331-490 e in da Barberino A. I Reali di Francia, a c. di Vandelli G., Gambarin G., Laterza, Bari, 1947. Del Libro di Fioravante, invece, è stata da me allestita un'edizione provvisoria con relativo commento in appendice alla mia tesi Il "Libro di Fioravante" cit., pp. 269-401. Il romanzo di Francia, infine, è stato oggetto specifico della tesi di dottorato, conseguito presso la Sorbona di Parigi, di Mc Arthur D, dal titolo Il Romanzo di Francia, une version du Libro di Fioravante édité d'après le manuscrit unique conservé à la Bibliothèque nationale. 
ché per la cronologia, discendono verosimilmente da un archetipo perduto risalente al XIV secolo, etichettato come "primo Libro di Fioravante". Tale exemplar, a sua volta, ha alle spalle una complessa tradizione, che fa capo, in ultima analisi, ad un poema originale francese perduto di metà XII secolo, che aveva come protagonista un eroe chiamato Floovent. ${ }^{4}$ Questa tradizione, sdoppiatasi in due rami principali, si è poi diffusa in Europa, fino ad essere nuovamente riunita al di qua delle Alpi.

I quattro testi italiani menzionati erano già stati segnalati, e i primi due editi, entro la prima metà del Novecento, e la situazione sembrava destinata a rimanere immutata. ${ }^{5}$ Oggi, tuttavia, il panorama degli studi appare assai diverso, sia per l'indagine avviata da Mc Arthur sul Romanzo di Francia, sia per l'edizione del Libro di Fioravante attualmente in cantiere, sia in quanto la lista delle compilazioni si è arricchita di una quinta versione di fattura italiana a lungo passata sotto silenzio. ${ }^{6}$ Il merito della scoperta di questa nuova redazione, indicata come Storia di Fioravante o Fioravante della Badia, va attribuito a Gianfranco Contini, che la rinvenne alcuni decenni or sono tra i manoscritti che un tempo appartenevano al Convento della Badia Fiorentina. ${ }^{7}$ I volumi conservati presso questa istituzione religiosa sono passati, in seguito alla soppressione, alla Biblioteca Nazionale Centrale di Firenze, e il codice

3 É lo stesso Mc Arthur D. a fissare la cronologia di questo archetipo, da lui definito, appunto, "premier Libro di Fioravante"; secondo il filologo, si tratterebbe di «une oeuvre italienne qui n'existait pas avant le XIVe siècle et qui ne devint pas célèbre qu'après 1350» (Mc Arthur D. Les versions cit., p. 122).

4 Per la formazione del poema primitivo perduto su Floovent, la cui genesi sarebbe in rapporto con i canti epici del cosiddetto ciclo merovingio, cfr. Darmesteter A. De Floovante vetustiore Gallico poemate cit., pp. 33-34; 95-105; 110; 113. Ho effettuato un tentativo di ricostruzione completa, sulla base degli studi precedenti, della tradizione europea scaturita dall'originale di metà XII secolo, unitamente ad una descrizione delle versioni non italiane della leggenda, nella mia tesi Il "Libro di Fioravante" cit., pp. 20-50.

5 A questo proposito, si ricordino, in particolare, Darmesteter A. De Floovante vetustiore Gallico poemate cit., pp. 69-87 e le rispettive edizioni in Rajna P. Ricerche cit., pp. 331-490 e in da Barberino A. Reali cit.

6 Cfr. la tesi di Mc Arthur D. su Il Romanzo di Francia e Mc Arthur D. Les versions cit.

7 La segnalazione di questo ritrovamento si deve, in prima istanza, a Bendinelli M.L. Preistoria dell'"Aiolfo" di Andrea da Barberino. Studi di filologia italiana 25, 1967, pp. 103-108: 103. 
che conserva la Storia di Fioravante reca attualmente la segnatura Conv. Soppr. da Ord. Badia 30. Il ritrovamento di Contini non ebbe un'eco rilevante nell'immediato, tanto che, ancora negli anni '70 del secolo scorso, Altamura, cui si deve un lavoro sistematico sul Romanzo di Francia, non conosceva che le quattro stesure precedentemente citate, sebbene già alcuni anni prima la Bendinelli avesse eseguito un breve ma significativo sondaggio sul Fioravante della Badia. ${ }^{8}$ Problematico, parimenti, è stato stabilire la natura di questo testo; la questione era ed è decidere se si tratti davvero di una distinta versione della vicenda di Fioravante o, piuttosto, se il codice che la trasmette sia un nuovo testimone di una delle altre redazioni. Il sospetto che ci si trovi di fronte ad un ulteriore testimone di un'altra redazione, in particolare del Libro delle Storie di Fioravante, è stato avanzato da Marco Villoresi, il quale ha creduto di riconoscere in esso un terzo manoscritto - addirittura «il più antico tra quelli conosciuti» - di questo romanzo cavalleresco trecentesco, insieme al ms. II II 28 della BNCF e al ms. Mediceo Palatino 119 della Biblioteca Medicea Laurenziana di Firenze, dei quali si era servito Rajna per l'edizione ottocentesca dell'opera.'

Per la prima soluzione, ossia per l'ammissione dell'esistenza di una quinta compilazione, tuttavia, sembrava già inclinare la Bendinelli che, pur consapevole delle affinità tra Il Libro delle Storie di Fioravante e la Storia di Fioravante, istituiva un utile confronto tra di essi (e con il Libro di Fioravante) in base al quale si potrebbe affermare che essi sono testi simili, ma non del tutto coincidenti. ${ }^{10}$ Questa idea è ribadita, in maniera indiretta, anche dalla Delcorno Branca, la quale considera il Fioravante della Badia come una nuova versione delle vicissitudini del cavaliere francese trasmessa da un testimone unico. ${ }^{11}$

8 Altamura A. Il "Romanzo di Francia", in Studi di filologia italiana, XI, Fiorentino, Napoli, 1972, pp. 67-79: 70, infatti, non dà traccia di questa quinta versione. Il sondaggio della Bendinelli è materia del già citato Preistoria dell' "Aiolfo".

9 Villoresi M. La tradizione manoscritta dei testi cavallereschi in volgare. Cantari, poemi, romanzi in prosa, in Intorno al testo. Tipologie del corredo esegetico e soluzioni editoriali. Atti del Convegno di Urbino, 1-3 Ottobre 2001, Salerno editrice, Roma, 2003, pp. 143-172: 161, n. 29, poi in Villoresi M. La fabbrica dei cavalieri. Cantari, poemi, romanzi in prosa fra Medioevo e Rinascimento. Salerno Editrice, Roma, 2005, pp. 11-37: 27, n. 26 (da cui si cita).

10 Bendinelli M.L. Preistoria dell'“Aiolfo" cit., pp. 103; 105-108.

11 Delcorno Branca D. Il romanzo cavalleresco medievale. Sansoni, Firenze, 1974 , p. 18 
Il confronto istituito dalla Bendinelli era limitato ai capitoli iniziale e finale della Storia di Fioravante, raffrontati con le parti corrispettive del Libro delle Storie di Fioravante di Firenze curato da Rajna e con il Libro di Fioravante copiato a Pontevico nel 1467 e tràdito dal ms. it. 1647 della Bibliothèque Nationale di Parigi. A prescindere dai Reali di Francia e dal Romanzo di Francia - opere nelle quali la capacità rielaborativa e inventiva di chi le ha confezionate ha agito incondizionatamente, tanto che solo occasionalmente si rivelano qui utili termini di paragone -, sembra opportuno effettuare una più ampia comparazione tra le altre tre compilazioni al fine di dimostrare che, seppure imparentate fra loro, esse sono tre opere distinte della tradizione letteraria italiana. ${ }^{12}$ Prima di procedere al raffronto puntuale tra alcuni passi significativi delle tre redazioni vagliate, si forniscono alcuni dati essenziali su ciascuna di esse.

Il Libro delle Storie di Fioravante è probabilmente la versione più antica che discende dal supposto "primo Libro di Fioravante". Il romanzo in questione è anonimo e ci è giunto attraverso due codici fiorentini, i già citati ms. II II 28 delle BNCF e ms. Mediceo Palatino 119 della BMLF, entrambi miscellanei e scritti in volgare toscano; in essi, il testo relativo a Fioravante occupa, rispettivamente, i ff. $51 r$ - $92 r$ e i ff. $7 r$ $48 v \cdot{ }^{13}$ I due volumi furono collazionati da Rajna per l'edizione dell'ope$\mathrm{ra}$, che si trova in appendice alle sue illustri Ricerche intorno ai "Reali di Francia" del 1872. Lo studioso, in seguito ad un serrato confronto lessicale e stilistico con i Reali barberiniani, riteneva di assegnare la composizione del Libro delle Storie di Fioravante al periodo 1315-1340, anche se entrambi i testimoni che lo conservano sono ascrivibili al pieno Quattrocento. ${ }^{14}$

12 Il Libro delle Storie di Fioravante e i primi III libri dei Reali di Francia, nei quali è narrata la leggenda di Fioravante corrispondente, sebbene con notevoli libertà, alle altre redazioni analizzate, sono già stati messi ampiamente a confronto in Rajna P. Ricerche cit., pp. 7-113.

13 Informazioni puntuali sui due manoscritti che trasmettono Il Libro delle Storie di Fioravante si trovano in Rajna P. Ricerche cit., pp. VIII-XII, Darmesteter A. De Floovante vetustiore Gallico poemate cit., p. 46, Andolf S. Floovant cit., p. LV, Villoresi M. La letteratura cavalleresca. Dai cicli medievali all'Ariosto, Carocci, Roma, 2000, p. 63 e Villoresi M. La tradizione manoscritta cit., p. 161.

14 Rajna, in particolare, stimava che l'intervallo tra la composizione del Fioravante di cui si stava occupando e l'ideazione del proprio romanzo da parte di maestro Andrea fosse di almeno cinquant'anni (cfr. Rajna P. Ricerche cit., p. 33). Questa 
Sempre nel XV secolo è stato confezionato il manoscritto unico che tramanda la Storia di Fioravante, il Cod. Conv. Soppr. da Ord. Badia $30 .{ }^{15}$ Anche in questo caso il testo è scritto in un volgare prettamente toscano e si estende per i 56 fogli che costituiscono il volume, precisamente dal f. $1 r$ al f. $56 r$ (ma sono bianchi i ff. $44 v-45 r$ ). Nella parte inferiore dell'ultimo foglio è presente una nota, parzialmente erasa, che consente di stabilire con esattezza la datazione dell'opera e, con un certo margine di errore, anche l'identità del copista; si legge, infatti, che «Questo libro fue finito di scrivere questo dì XV di giugno 1464 e.ffue scritto per-lle mani di Miniato di $\mathrm{B}$ [an]co richattiere. Preghiamo Iddio che chi lo scrisse e chi lo lege possa vivere e morire nello amore di Gesù Cristo». ${ }^{16}$ Se nessun dubbio sembra sussistere per la cronologia di questa copia, qualche problema è suscitato dal nome dell'amanuense che l'ha realizzata, dato che esso non è chiaramente comprensibile. A questo riguardo, la Bendinelli ha suggerito la lezione alternativa «Miniato d'Antonio Achattioni». ${ }^{17}$ La questione onomastica può forse essere risolta per via congetturale a partire dalla nota di possesso autografa collocata in apertura del codice, che stabilisce che «Questo libro è di Piero di Simone del Nero donatomi da messer Piero Cambi». Piero del Nero fu erudito e bibliofilo cinquecentesco, i cui antenati, quando si insediarono nel capoluogo toscano, esercitarono la modesta professione di rigattieri. ${ }^{18}$ Si potrebbe supporre, quindi, che il

ricostruzione cronologica ha trovato l'accordo della Mattaini (Romanzi dei Reali di Francia, a c. di Mattaini A., Rizzoli, Milano, 1957, p. 12), mentre in maniera più generica Mc Arthur D. Les versions cit., pp. 122; 124 riconduce Il Libro delle Storie di Fioravante alla prima metà del XIV secolo e i Reali al Quattrocento.

15 Una descrizione paleografica del volume in questione è fornita da I manoscritti datati del fondo Conventi Soppressi della Biblioteca Nazionale Centrale di Firenze, a c. di Bianchi S. e altri, SISMEL - Edizioni del Galluzzo, Firenze, 2002, p. 116.

16 Bianchi S. I manoscritti datati cit., p. 116.

17 Bendinelli M.L. Preistoria dell'“Aiolfo" cit., p. 105.

18 Un'accurata descrizione della vita e della biblioteca di Piero o Pietro del Nero si ha in Gregori L. Piero del Nero tra bibliofilia e filologia. Aevum 62, maggio-agosto 1988, pp. 316-361 e Gregori L. I codici di Piero Del Nero negli spogli lessicali della Crusca. Aevum 64, settembre-dicembre 1990, pp. 375-385. Informazioni dettagliate sulle attività dei rigattieri, dei sarti e dei tessitori di lino si trovano in Davidsohn R. Storia di Firenze, IV. II, Sansoni, Firenze, 1965, pp. 97-99; 162-163; 324-329. In particolare, la corporazione dei linaiuoli (che includeva anche i pennaioli), avendo rifiutato di aggregarsi a una delle arti maggiori quando furono emanati gli Ordinamenti di 
volume che conserva la Storia di Fioravante sia stato prodotto in questo ambiente sociale da un rigattiere, tale Miniato di Banco, che si dedicava alla letteratura a tempo perso, e che lì abbia continuato a circolare fino a giungere nelle mani di Piero del Nero.

Più o meno agli stessi anni, ma ad una diversa area geografica, è riconducibile la terza stesura in esame, ossia il Libro di Fioravante del ms. it. 1647 della Bibliothèque Nationale di Parigi. ${ }^{19}$ L'opera, anonima, occupa tutti i 60 fogli del codice e mostra una certa commistione linguistica; è scritta, infatti, in un volgare settentrionale (con una netta prevalenza di tratti veneti e lombardi) frammisto di elementi toscaneggianti e gallicheggianti. Anche in questo caso una nota posta in chiusura del testo permette di conoscere con certezza la data e il luogo di compilazione del volume: «Finito el libro de Fioravante nel anno $\mathrm{M}^{\circ} \mathrm{CCCC}^{\circ} \mathrm{LXVII}$ a dì X de settembre in la rocca de Pontevigo», località tuttora esistente (Pontevico) al limitare della Provincia di Brescia. ${ }^{20}$

Le versioni che presentano le maggiori analogie a livello testuale sono il Libro delle Storie di Fioravante (F) e la Storia di Fioravante (B); il Fioravante fiorentino, tuttavia, condivide occasionalmente alcuni dettagli con il Libro di Fioravante di Pontevico (P), che, viceversa, non compaiono nel testo scoperto da Contini. Quest'ultimo romanzo, d'altronde, si lega al precedente per certe peculiarità sconosciute alla prosa edita da Rajna.

Già ad una prima osservazione si rileva che il Libro delle Storie di Fioravante si presenta suddiviso in capitoli, ciascuno dei quali è preceduto da una rubrica che ne sintetizza l'argomento; quella di esordio, in

giustizia, fu assolutamente autonoma fino al 1300. A partire da quest'anno, tuttavia, si unì ai rigattieri, che, proprio in virtù degli Ordinamenti, erano divenuti da Arte media un'Arte maggiore. I rigattieri, che commerciavano vecchi vestiti e pellicce, avevano le loro botteghe soprattutto nelle zone del Mercato Vecchio e del Mercato Nuovo. Uno studio di carattere generale sul sistema corporativo fiorentino è Gandi G. Le corporazioni dell' Antica Firenze. Edito a cura della Conf. Naz. Fascista dei Commercianti, Firenze-Roma, 1928; in particolare, a pp. 225-229 del libro si parla dell'Arte dei Linaioli e Rigattieri.

19 Tra i primi ad interessarsi a questo manoscritto vi fu Mazzatinti G., che ne fornì una descrizione codicologica e ne trascrisse alcuni fogli (Inventario dei manoscritti italiani delle Biblioteche di Francia, II, Fratelli Bencini, Roma, 1887, pp. 543-548).

20 Si segnala che nella scheda di Mazzatinti G. Inventario dei manoscritti italia$n i$ cit., l'anno viene trascritto erroneamente come $\mathrm{M}^{\circ} \mathrm{CCC}^{\circ}$ lxvij, cosicché la cronologia del codice risulta anticipata di un secolo. 
particolare, recita: «Qui comincia il libro di Fioravante e sua nazione, come narrano ne' ramanzi le loro storie». ${ }^{21}$ Nelle altre due redazioni, invece, la sola rubrica che si trova è posta in apertura dell'intera prosa; nella Storia di Fioravante si legge «Al nome sia de Dio e della sua Madre Virgine Maria e del beato messer Santo Giovanni Batista Evangelista e di tutta la santa corte del Paradiso incominceremo da qui nui inazi a scrivere tutta la storia di Fioravante, il quale si.ffu figliuolo del re di Francia e.ffu chiamato lo re Fiore», mentre nel Libro di Fioravante è scritto semplicemente «Questo si è el libro de Fioravante cavalero e de Dusolina soa moiere».22

Segue, in tutti e tre i casi, la sezione iniziale del romanzo, dedicata alle gesta del nonno di Fioravante. Si nota immediatamente una differenza onomastica tra le tre compilazioni, in quanto nel Fioravante di Firenze il personaggio in questione è chiamato Fiovo, mentre nelle altre due stesure viene chiamato, rispettivamente, Fiore e, con una lieve variazione, Fiorio. ${ }^{23}$ Riteniamo che la prima forma sia maggiormente vicina all'originale, ossia al nome che si poteva trovare all'altezza del "primo Libro di Fioravante" o di una sua copia successiva, in quanto essa sarebbe l'esito finale di un'evoluzione fonetica dal francese Flovent. ${ }^{24}$ Solo in una fase posteriore, presumibilmente, si sono generate due versioni toscane della storia, in una delle quali il nome è rimasto

21 Rajna P. Ricerche cit., p. 333. Si avverte che, per effettuare i confronti successivi tra le tre versioni della vicenda del cavaliere francese, ci si è serviti de Il Libro delle Storie di Fioravante pubblicato da Rajna P.

22 Le due rubriche sono trascritte, rispettivamente, dal f. $1 r$ del Cod. Conv. Soppr. da Ord. Badia 30 e dal f. $1 r$ del ms. it. 1647. Per il testo tràdito da quest'ultimo codice, l'edizione provvisoria di riferimento è quella da me allestita in appendice nella mia tesi Il "Libro di Fioravante" cit., pp. 271-346.

23 A proposito dei nomi, si avverte che, qualora non ci sia completo accordo su di essi nelle tre redazioni in esame, saranno riportate le forme differenti di ciascuna secondo la sequenza F/B/P. Nel caso ci siano solo due forme onomastiche alternative, esse sono ascrivibili, rispettivamente, alla coppia F-B e a P.

24 Quest'ultimo appellativo sarebbe stato attribuito a Floovent in uno dei due rami $(\beta)$ della tradizione europea, in seguito ad una contrazione vocalica. A contatto con i volgari italiani si sarebbe avuta, poi, un'ulteriore modificazione fonetica, sulla quale Darmesteter così si esprime: «Fiovo vocatur qui in islandico Flovent; Fiovo et Flovent unum ac idem esse nomen facile apparet; Flovent enim in italiano sermone Fiovente sonat» (cfr. Darmesteter A. De Floovante vetustiore Gallico poemate cit., p. 33). In altri termini, si sarebbero verificati i seguenti passaggi: Floovent $>$ Flovent $>$ Fiovente $>$ Fiovo. 
Fiovo, mentre nell'altra è stato trasformato in Fiore, poi ulteriormente modificato in Fiorio nell'Italia del Nord. ${ }^{25}$

Procedendo oltre le divergenze onomastiche, si può constatare che mutano decisamente anche l'estensione e i contenuti della prima parte delle tre opere. Le avventure di Fiovo, infatti, occupano i primi XVI capitoli del testo curato da Rajna, che sono così riassumibili: ${ }^{26}$

(Cap. I) Fiovo, che si trova alla corte di Roma, versa accidentalmente una coppa sul mantello di un "turchio", che lo insulta e lo colpisce. Il giovane impulsivamente lo uccide ed è costretto a scappare insieme ai cugini Gifroi e Otto. Gostantino li insegue, ma Fiovo lo disarciona e lo ferisce; poi, i tre fuggitivi giungono presso un eremita alla montagna di Radicofani. Il romito, saputo da un angelo che è proprio Fiovo ad aver bussato alla sua porta, lo accoglie, gli dona delle armi, si confessa da lui e muore serenamente. I tre cavalieri riprendono il viaggio e, presso la Lombardia, si scontrano con dodici ladroni saracini, tre dei quali riescono a fuggire. Fiovo e i suoi compagni arrivano ad un castello dove trovano ospitalità fingendo di non essere cristiani, ma vengono scoperti dai tre ladri sopravvissuti e fuggono di nuovo. Arrivati in un bosco, incontrano Ansuigi, loro parente. In seguito, Fiovo caccia una cerva e per questo motivo sfida e uccide un saraceno. Gli amici di quest'ultimo lottano con Fiovo e i suoi, ma vengono sconfitti. Poi Fiovo, Ansuigi, Gifroi e Otto vanno a Provino, dove si fanno reclutare come cavalieri per soccorrere il re Fiorenze, assediato a Parigi da Salatres. Giunti a Parigi, i quattro restano una notte fuori dalla città e il giorno dopo combattono contro trecento saraceni e li vincono. (Cap. II) Il capitano turco Corsabrino, udendo ciò, arma mille cavalieri e cattura Otto; intanto, anche re Fiorenze esce in battaglia per aiutare Fiovo. Quest'ultimo batte Corsabrino, che consiglia a Salatres di ritirarsi. (Cap. III) Fiovo torna vittorioso a Parigi e viene nominato capitano di guerra, ma è addolorato perché Otto è ancora prigioniero dei saraceni. (Cap. IV) Brandoia, figlia di Salatres, ha in custodia Otto e crede che sia Fiovo ma, venuta a conoscenza della verità, gli chiede di andare dal vero Fiovo promettendo di divenire cri-

25 Sul diverso nome di questo personaggio rispetto alla redazione del Libro delle Storie di Fioravante (e ai Reali di Francia) è intervenuto Rajna P., per il quale «Il "Fiorio" del Fioravante di Parigi [...] sarà stato probabilmente in origine una mera corruzione di scrittura. Ma esso si trovò ribadito dall'opportunità di distinguere il figlio dal padre, non più "Fiovo", ma "Fiorio" ancor lui» (Rajna P. Avanzi di una versione toscana del "Buovo d'Antona". Zeitschrift für romanische Philologie 12, 1888, pp. 463-510: 470, n. 6). Non si può escludere, tuttavia, un'influenza sulla forma "Fiorio" del nome del protagonista del trecentesco Cantare di Fiorio e Biancifiore, assai vulgato in area settentrionale.

26 Rajna P. Ricerche cit., pp. 333-366. 
stiana se egli la vuole come moglie. Frattanto Corsabrino invia dei doni di nascosto a Fiovo insieme a una lettera, chiedendogli di prepararsi per la guerra. (Cap. V) Brandoia chiede a Corsabrino di condurre nottetempo Otto a Parigi, affinché domandi a Fiovo di sposarla. Fiovo accetta e in circa un mese raccoglie una grande armata, con la quale va a prendere Brandoia. (Cap. VI) Salatres scopre che la figlia è stata rapita e, su consiglio di Corsabrino, sfida Fiovo alla battaglia. Vengono preparate le schiere e inizia lo scontro; Salatres e i suoi soldati vengono messi in fuga, (Cap. VII) ma il re saracino, consigliato dai propri baroni, ricostituisce le truppe. Corsabrino avverte Fiovo, che propone a re Fiorenze di fare un'ambasciata a Roma per domandare aiuto. Otto si reca da Gostantino di nascosto e gli racconta quanto è successo, poi l'imperatore accoglie gli ambasciatori e promette loro diecimila cavalieri. (Cap. VIII) Gli ambasciatori restano a Roma intanto che Gostantino raduna la propria gente, poi tornano a Parigi. (Cap. IX) Corsabrino dice a Salatres che Fiovo ha chiesto soccorso a Gostantino e suggerisce di mandare Dinasor ad assediare Roma. Intanto, i cristiani arrivano a Parigi e c'è una nuova battaglia fra gli eserciti di Fiovo e Salatres, che viene ucciso. (Cap. X) Fiovo sposa Brandoia, anche se re Fiorenze avrebbe voluto dargli in moglie la propria figlia. Quest'ultima cerca di avvelenare Fiovo, che, scoperto il fatto, uccide re Fiorenze e impone a tutti il battesimo. (Cap. XI) Fiovo conquista la Francia, la Scozia, l'Irlanda e molti altri Paesi, poi assegna a Corsabrino il reame di Brettagna. (Cap. XII) La figlia di re Fiorenze chiede grazia a Fiovo, si converte e viene data in sposa ad Ansoigi. Da lei ha origine la stirpe dei traditori di Maganza. (Cap. XIII) Un messo dice a Fiovo che Gostantino è assediato e il nuovo re di Francia si dirige verso Roma. (Cap. XIV) Fiovo e Gostantino sfidano Dinasor alla battaglia, ma i cristiani vengono messi in fuga. Un villano, vedendo ciò, decide di scontrarsi con Dinasor e lo cattura. I saracini vengono poi sconfitti e il villano viene onorato da Gostantino. (Cap. XV) Quest'ultimo chiede a Dinasor di convertirsi, ma egli rifiuta e muore in prigione. (Cap. XVI) Dopo circa un mese, Fiovo torna in Francia, dove Brandoia gli genera due figli maschi: Fiorello e Fiorio. Gostantino lo chiama poi a Roma per ricevere la corona dell'impero. Fiovo incorona Fiorello del reame di Francia e gli dà per moglie Bianciadore, figlia del re della Magna, mentre Fiorio sposa la figlia del re di Dardenna e ne eredita il regno. Poi Fiovo va a Roma e qui muoiono sia lui che Gostantino.

Questa prolungata sezione incipitaria si chiude con la dichiarazione del narratore che da questo punto in avanti saranno narrate le vicende di Fioravante.

Il Fioravante della Badia e il Libro di Fioravante del ms. it. 1647 riservano entrambi i ff. $1 r-2 r$ al racconto delle imprese di Fiore/Fiorio, che si svolgono in maniera pressoché identica, fatti salvi alcuni cambiamenti di tipo onomastico, nonché nella scelta dei vocaboli e nell'orga- 
nizzazione sintattica delle singole proposizioni, in base al principio già enunciato dalla Bendinelli che «il testo lombardo [il Libro di Fioravante] condensa spesso le frasi narrativamente indugiate del toscano [la Storia di Fioravante $] \gg .{ }^{27}$ Ecco una breve sintesi di questa sezione comune alle due prose suddette:

Fiore/Fiorio viene bandito da Roma da Gostantino/Constantino per aver ucciso un uomo (senza ulteriori precisazioni). Insieme al nipote Salino/Sufficiente si reca in Francia, a Proino/Polunio, e qui sposa la saracena convertitasi Brandina/Biondora la Bella. Fiore/Fiorio raduna centomila cavalieri e va ad assediare Parigi, dove si trova re Firenze/Fiorenzo. Quest'ultimo si scontra con Fiore/Fiorio, che sembra avere la peggio. Brandina/Biondora accorre in suo aiuto e insieme uccidono re Firenze/Fiorenzo. La moglie di questi viene data in sposa a Salino/Ansuixi (variazione rilevante del codice di Pontevico, in quanto introduce un nuovo personaggio) e da loro ha origine la casa di Maganza. Fiore/Fiorio viene incoronato del reame di Francia e gli nascono i figli Fiorello e Fiovo/Fionio. L'imperatore Gostantino/Constantino, assediato dai saracini, chiede soccorso a Fiore/Fiorio, promettendogli in cambio il perdono e la pace. Fiore/Fiorio accetta e in un anno raduna le proprie truppe, poi va a liberare Roma. Gostantino/Constantino muore e a Fiore/Fiorio viene data la corona imperiale. Brandina/Biondora manda i figli a conquistarsi dei regni: Fiovo/Fionio si propone di ottenere la mano della figlia del re di Dardana, mentre Fiorello sposa Bianciadore/Bianzadore, figlia del re della Magna, e ottiene la Francia.

É evidente, dunque, che c'è una concordanza quasi assoluta, a livello di contenuto, tra le ultime due redazioni considerate, mentre $I l$ Libro delle Storie di Fioravante risulta assai distante da esse. Dato che i casi di Fiovo sono narrati ampiamente, sebbene con variazioni, anche nel Libro I dei Reali di Francia e nel Romanzo di Francia, è plausibile che essi fossero leggibili nell'exemplar della tradizione italiana e che da qui siano passati nel testo primitivo, verosimilmente toscano, che è servito come fonte sia per la versione napoletana che per quella edita da Rajna. ${ }^{28}$ Quest'ultima, poi, avrebbe costituito il modello diretto per il racconto su Fiovo inserito nel romanzo barberiniano.

27 Bendinelli M.L. Preistoria dell'“Aiolfo" cit., p. 103.

28 Per la parte relativa a Fiovo ne I Reali di Francia e ne Il Romanzo di Francia, cfr., rispettivamente, da Barberino A. Reali cit, Libro I, capp. IV-XLII, LIV-LIX, pp. 7 76; 95-111 e Altamura A. Il "Romanzo di Francia" cit., p. 72. 
Addentrandosi nella parte riservata specificamente a Fioravante, che rappresenta in tutti e tre le stesure la porzione testuale maggiore, si coglie subito un elemento che accomuna, questa volta, F e P: rispettivamente al Cap. XVII e al f. $2 v$, si dice, infatti, che l'eroe si reca dal padre, il re Fiorello, per domandargli armi per conquistarsi un regno all'età di nove anni. ${ }^{29}$ Questo particolare non è condiviso, invece, da B, in cui (f. $2 v$ ) il protagonista espone la propria richiesta al genitore raggiunti i diciassette anni. Si tratta, è vero, di un piccolo dettaglio, ma il fatto che si trovi sia in $\mathrm{F}$ che in $\mathrm{P}$ induce a credere sia stato attinto da una medesima fonte, poi alterata, per questo punto, in B.

Subito dopo, tuttavia, riprendono le analogie tra la prosa pubblicata da Rajna e quella rinvenuta da Contini a proposito del primo episodio di rilievo della vita di Fioravante, ossia il taglio della barba perpetrato a danno del maestro di scherma Salardo. In entrambi i testi, rispettivamente al Cap. XVII e al f. $3 r$, viene precisato che Salardo russa forte in quanto è vecchio e che il gesto irriverente del giovane cavaliere è causato dall' "iniquità".$^{30}$ In $\mathrm{P}(\mathrm{f} .2 v)$, al contrario, non viene fornita alcuna spiegazione del russare eccessivo del maestro Salandro né di che cosa spinga l'allievo a oltraggiare quest'ultimo.

In seguito all'episodio appena ricordato, Fioravante è costretto a lasciare Parigi e si imbatte in una donzella, Ulia, che salva prima da tre saraceni e poi dal pagano Farnagù/Ferraù/Feragù. Il padre di quest'ultimo, re Galerano/Gallerano/Gallarano, dopo aver fatto un terribile sogno, invia alcuni uomini alla ricerca del figlio e questi catturano Fioravante, il quale viene poi liberato dal paladino Riccieri/Ricieri/ Rizero. Si registrano qui delle differenze tra il Fioravante fiorentino da un lato e quello della Badia e di Pontevico dall'altro. Nella prima compilazione, ai Capp. XXII-XXIV, infatti, si parla di ben cinque pagani, fra cui tre re, che prendono Fioravante e che vengono uccisi, successivamente, da Riccieri, mentre Fernagù muore per mano di Fioravante stesso..$^{31}$ In $\mathrm{B}$ (ff. $6 r-7 v$ ) e in P (ff. $6 r-7 r$ ), invece, ad imprigionare Fioravante sono soltanto tre re saraceni; di essi due vengono colpiti mortalmente da Ricieri/Rizero - che uccide anche Ferraù/Feragù - e il terzo viene passato a fil di spada da Fioravante.

29 Rajna P. Ricerche cit., p. 366.

30 Rajna P. Ricerche cit., p. 367.

31 Rajna P. Ricerche cit., pp. 374-378. 
Nel prosieguo della narrazione, Fioravante, il paladino e Ulia si dirigono verso la città di Balda, ma vengono ingannati da un uomo di scarsa dignità sociale e morale, che addormenta i due cavalieri e rapisce la donzella. In F (Cap. XXVI) e in B (f. 8r), Fioravante e Riccieri/Ricieri si destano per effetto di una radice magica che fa loro muovere la testa fino a che la immergono nell'acqua (nel primo testo si parla di una fonte e nel secondo di una pozza), mentre in $\mathrm{P}$ ( $\mathrm{f} .7 v$ ) il merito dell'improvviso risveglio è attribuito unicamente alla radice, che Rizero fa ingoiare al proprio signore. ${ }^{32}$

Per un lungo tratto, poi, non si notano differenze sostanziali tra le tre redazioni in esame; le vicissitudini di Fioravante proseguono con la sua cattura ad opera dei saraceni, l'amore - corrisposto - di Drugiolina/Drusolina/Duxolina nei suoi confronti, la sua liberazione da parte dell'esercito cristiano, il rientro a Parigi, la fuga per evitare un matrimonio combinato, l'incontro con un eremita e, infine, l'arrivo a Scondia (Scorcia in $\mathrm{P}$ ), dove il soldano, pretendente respinto di Drugiolina/Drusolina/Duxolina, ha posto l'assedio. Qui ricominciano le discrepanze, seppure di modesta entità, tra il Libro delle Storie di Fioravante e la Storia di Fioravante da una parte e il Libro di Fioravante dall'altra. Nelle prime due stesure, innanzitutto, si legge (rispettivamente al Cap. XLVII e al f. 22r) che, quando Fioravante giunge nei pressi di Scondia, il soldano manda contro di lui sei cavalieri; il protagonista ne uccide repentinamente uno e mette in fuga gli altri cinque. ${ }^{33}$ Il numero degli uomini inviati a contrastare Fioravante, al contrario, non è indicato nella compilazione del ms. it. 1647 (f. 20v) ed essi, addirittura, spaventati dall'aspetto dell'eroe, si ritirano senza combattere.

In tutte e tre le versioni si passa, successivamente, allo scontro aperto con le truppe del soldano. Anche in questa circostanza, F (Cap. XLIX) e B (f. $23 v$ ) sono concordi nel citare, fra gli altri, un cavaliere che viene ucciso da Fioravante, tale Giraldone di Marmedonia/ Grialdone di Manzidonia, mentre il racconto di P (f. 22r) si mantiene su una linea molto più generale e il personaggio in questione non viene menzionato. ${ }^{34}$

Nelle scene seguenti non si denunciano particolari divergenze:

32 Rajna P. Ricerche cit., p. 379.

33 Rajna P. Ricerche cit., pp. 412-413.

34 Rajna P. Ricerche cit., p. 416. 
Fioravante sconfigge il soldano e, sotto mentite spoglie, vive alla corte di re Balante, padre di Drugiolina/Drusolina/Duxolina. Smascherato da un buffone, viene imprigionato e scappa grazie all'ausilio della donna amata. I due si rifugiano, quindi, in un castello custodito da donne, che in F (Cap. LV) e in B (f. 33r) dichiarano di avere scorte sufficienti alcune per più di dieci, altre per più di sette anni, mentre in $\mathrm{P}$ (f. $29 v$ ) solo per due o, addirittura, per un solo anno. ${ }^{35}$ Ciò farebbe propendere per la teoria che, per l'intero episodio del castello, le redazioni di $\mathrm{F}$ e di B coincidano, a scapito di quella di $\mathrm{P}$, verosimilmente modificata. Non è, tuttavia, così; nella prima versione, infatti, il castello in cui Fioravante e Drugiolina sono trattenuti è chiamato Mongrifalco (Cap. LVII), mentre nelle altre due si ha, rispettivamente, il nome, diverso ma assai simile, Moltalto (f. 35r) e Monte Alto (f. 31r). ${ }^{36}$ Si può supporre che un primo cambiamento onomastico sia avvenuto tra $\mathrm{F}$ e $\mathrm{B}$, versioni che avrebbero alle spalle un medesimo archetipo, e che poi la forma Moltalto abbia subito un'ulteriore trasformazione fino a giungere a quella leggibile nel codice copiato a Pontevico.

Una volta libero, in tutti e tre i romanzi, Fioravante torna a Parigi con la propria amata e la sposa. Dopo sette anni, ella partorisce due gemelli, Gisberto/Zilberto e Attaviano/Octaviano ma, a causa delle calunnie della regina madre, viene esiliata con i figli. Uno dei bambini viene poi sottratto da un gigante e l'altro da un leone. A proposito di questi due episodi si rilevano differenze più o meno cospicue che, ancora una volta, oppongono il Fioravante di Firenze e quello della Badia al Libro di Fioravante del manoscritto di Parigi. Nel caso del gigante, si tratta di un dettaglio di limitata importanza: nelle prime due prose (rispettivamente al Cap. LXIII e al f. 40r), questo personaggio si scontra con gli uomini di un castellano suo nemico, il quale prende poi in custodia il fanciullo rapito. ${ }^{37} \mathrm{Nel}$ testo di Pontevico (al f. $37 r$ ), invece, il castellano soccombe durante la lotta con il "Zigante" e il bambino viene recuperato da un altro cavaliere.

Più problematica è la questione relativa al secondo rapimento, in quanto, a questo riguardo, la narrazione di $\mathrm{P}$ è assai dilatata e, a tratti, confusa, il che suggerisce che essa sia il frutto di un notevole rimaneg-

35 Rajna P. Ricerche cit., p. 436.

36 Rajna P. Ricerche cit., p. 440.

37 Rajna P. Ricerche cit., p. 452. 
giamento dovuto o al copista del ms. it. 1647 o a chi ha redatto una versione settentrionale antecedente del Libro di Fioravante a partire da una stesura presumibilmente toscana. D'altro canto, il testo di Pontevico sembra correggere un passaggio illogico che si riscontra nelle altre due redazioni, le quali collimano fra loro. Nel Libro delle Storie di Fioravante e nella Storia di Fioravante, infatti, rispettivamente al Cap. LXII e al f. 40r, si dice che Attaviano, il secondo dei figli di Drugiolina/Drusolina, viene preso da un leone, nel quale, in realtà, si è incarnato S. Marco. ${ }^{38} \mathrm{Il}$ racconto di questo rapimento viene poi interrotto per seguire le vicissitudini dell'altro gemello e riprende solo ai Capp. LXVIII-LXIX di F. e ai ff. 46r-47v di B. Quando in essi si torna a parlare di Attaviano, tuttavia, egli non risulta più essere stato sottratto alla madre da un leone, bensì da un grifone..$^{39} \mathrm{Il}$ primo animale entra in scena subito dopo e uccide il secondo. Alla vista di ciò, Drugiolina/Drusolina, spaventata, si rifugia su una nave, ma il leone, dopo aver nutrito il fanciullo con il proprio sangue, lo depone sulla riva del mare. La donna chiede ai marinai di tornare indietro per recuperare il figlio e, una volta giunti a riva, il leone sale a forza sull'imbarcazione per proteggere il bambino e sua madre, sventando un tentativo di violenza nei confronti di quest'ultima compiuto da un addetto alle scritture mercantili.

La compilazione conservata a Parigi, al contrario, mostra coerenza relativamente all'esecutore del rapimento: a f. $37 r$ si dice che Octaviano viene ghermito da un leone e che Duxolina lo insegue per la foresta, e questa è la situazione che si ritrova alla ripresa del racconto (ff. 42r-46r). L'animale, poi, si ferma e restituisce l'infante alla donna, ma dal cielo scende un grifone che afferra sia Octaviano che il leone e li porta su un'alta montagna. Duxolina sale a bordo di una nave saracena per raggiungere quel luogo e, una volta arrivata, il leone le riconsegna il fanciullo e si siede accanto a lei. La donna, successivamente, vede il grifone allontanarsi e torna sulla barca. Dato che i marinai rifiutano di far salire il leone, quest'ultimo segue la nave e vi salta dentro dopo che è stata levata l'ancora. Il padrone della nave attenta alla virtù di Duxolina, ma viene prontamente ucciso dal leone. ${ }^{40}$ Si potrebbe pen-

38 Rajna P. Ricerche cit., p. 452.

39 Rajna P. Ricerche cit., p. 462.

40 La presenza dell'animale nella redazione del ms. it. 1647 assume particolari 
sare che la sostituzione dell'addetto alle scritture mercantili con il padrone della nave sia un'invenzione, rispetto alle prose toscane, del redattore della versione settentrionale o dell'amanuense di Pontevico. C'è, però, un punto critico in questa congettura, dal momento che anche nei Reali di Francia (Libro II, cap. XLVIII) il padrone sostituisce lo scrivano. ${ }^{41}$ Dato che, soprattutto per il Libro II, maestro Andrea sembra avere tenuto presente il Fioravante fiorentino, non è escluso che nel "primo Libro di Fioravante" o in uno stadio assai prossimo ad esso comparisse lo scrivano, che sarebbe stato poi sostituito con il padrone nel romanzo barberiniano. Quando, infine, la storia di Fioravante è passata nel Nord della penisola, l'esecutore della versione settentrionale potrebbe aver mutuato questo elemento direttamente dai Reali, assai famosi sin dal primo XV secolo.

Le vicende di Attaviano/Octaviano, ad ogni modo, continuano in maniera abbastanza simile nelle tre redazioni in esame, fino a quando il personaggio, ormai adulto, libera - come aveva fatto il padre - re Balante dall'assedio del soldano e sposa la figlia di quest'ultimo. F e B inseriscono, a questo punto, un episodio che risulta, invece, assente in P. Nelle prime due stesure, infatti, rispettivamente al Cap. LXXIII e ai ff. 49r-50r, si narra che il soldano, pentitosi di aver dato in moglie la propria figlia ad Attaviano, tenta di avvelenarlo con la complicità di una cameriera, ma il piano viene sventato dal leone/S. Marco, mentre nel ms. it. 1647 (f. $47 v$ ) si parla solo del matrimonio di Octaviano. ${ }^{42}$

In tutte e tre le opere, poi, Drugiolina/Drusolina/Duxolina e il figlio tornano in Francia con re Balante, che pone l'assedio a Parigi. Gisberto/Zilberto, nel frattempo scelto come successore da Fioravante, e Attaviano/Octaviano si scontrano come campioni dei rispettivi eserciti, ma il leone/S. Marco rivela che essi sono fratelli e denuncia le calunnie della regina madre. Fioravante si riconcilia con i propri familiari e muore. Vengono esposte, quindi, le avventure separate dei suoi due eredi e, a questo riguardo, il Fioravante della Badia presenta una

implicazioni. Il copista di Pontevico, infatti, potrebbe avere inteso celebrare unitamente la religione cristiana e la Repubblica di Venezia, entrambe rappresentate dal leone/S. Marco, mediante la funzione salvifica assegnata al personaggio in questo passo e più avanti nella narrazione (f. $49 v$ ). Sulla questione, si veda Barbieri N.I. Un romanzo cavalleresco cit., pp. 36-37.

41 Cfr. da Barberino A. Reali cit., Libro II, cap. XLVIII, pp. 222-223.

42 Rajna P. Ricerche cit., pp. 469-470. 
notevole lacuna. Non perfetta, inoltre, è la coincidenza testuale tra la compilazione edita da Rajna e quella copiata a Pontevico. Sia nel Libro delle Storie di Fioravante sia nel Libro di Fioravante (rispettivamente ai Capp. LXXXI-LXXXIII e ai ff. 52v-53v), infatti, nell'ambito delle guerre in Oriente di Attaviano/Octaviano, si descrive lo scontro tra quest'ultimo e il re Daneburro/Naboro. Attaviano/Octaviano, poi, conquista il Santo Sepolcro e ha un figlio, che in F ha nome Bovetto - e del quale viene immediatamente enunciata la genealogia -, mentre in $\mathrm{P}$ viene chiamato Alban. ${ }^{43}$ In B tutta questa parte manca: la storia di Attaviano si interrompe improvvisamente al f. $55 \mathrm{r}$ e solo più tardi si parla di suo figlio, Bovetto (non nominato in precedenza), che, ancora impegnato militarmente nel Mediterraneo orientale, viene soccorso da Gisberto.

$\mathrm{Si}$ è accennato al fatto che nel tessuto narrativo del Fioravante di Firenze è inserita la genealogia di Attaviano. Nel capitolo conclusivo di questa redazione, il LXXXVII, trova posto anche l'elenco dei successori di Gisberto sul trono di Francia. La discendenza diretta del personaggio si esaurisce subito con Agnolo Michele, dopodiché il potere viene assunto dal siniscalco del regno, da cui nasce il re Pipino, padre di Carlo Magno; quest'ultimo, a propria volta, genera Aluigi e Aluzia, madre di Aiolfo. ${ }^{44}$ Questa ricostruzione dinastica non trova riscontro nelle altre due stesure, in quanto in entrambe (rispettivamente a f. 56r e a f. 55r) si crea una continuità fittizia tra la stirpe di Fioravante e i Carolingi, dicendo che Pipino/Pupino è nato da Agnolo Michele/Anzelo Micaele. L'identità tra le due compilazioni, tuttavia, si arresta qui, dal momento che la prosecuzione della schiatta reale francese leggibile ne La Storia di Fioravante è diversa rispetto a quella del Libro di Fioravante. ${ }^{45}$

Quest'ultimo romanzo, infine, si distingue nettamente dagli altri

43 Rajna P. Ricerche cit., pp. 482-486.

44 Rajna P. Ricerche cit., pp. 489-490.

45 Nel codice della Badia si ha la seguente discendenza: Gisberto, Agnolo Michele, Pipino, Carlo Magno, Luigi, Carlo Martello e Lottieri, con cui i sovrani francesi perdono la corona imperiale. Nel volume di Pontevico, invece, si dice che, dopo Zilberto, Anzelo Micaele e Pupino, il regno passa temporaneamente nelle mani di Lanfroi e Landrixe, ma viene poi riconquistato da Carlo Magno. I suoi eredi sono Alovixo, Carlo Martello, Carlo Mal Martello e, da ultimo, Elothe e Alothe, che causano il trasferimento dei favori del Pontefice dalla Francia ai principi tedeschi. 
due considerati - e da tutto il resto della tradizione, tanto italiana quanto europea - per la presenza di una "giunta", che occupa i ff. $55 v-60 v$ del ms. it. 1647, in cui si narra, dopo l'esposizione delle genealogie di Zilberto e Octaviano, il passaggio del potere imperiale dalla casa reale francese ai principi tedeschi. Essa, pertanto, qualifica in maniera specifica il testo custodito a Parigi e, in base ad elementi interni, può essere datata tra il 1452 ed il 1467, il che significa che poteva già trovarsi nel modello a disposizione del copista di Pontevico o che è stato proprio quest'ultimo a comporla. ${ }^{46}$

Al termine dell'analisi contrastiva eseguita, conviene trarre alcune conclusioni, tentando di chiarire i rapporti esistenti tra i tre romanzi vagliati e, secondariamente, cercando di ricostruire le relazioni tra questi ultimi e gli altri testimoni della propaggine italiana dell'epos di Floovent.

Una breve considerazione sulla qualità e le caratteristiche delle singole prose: il Fioravante curato da Rajna denuncia la propria arcaicità soprattutto nella carenza di sintesi e nella tendenza alla resa drammatica dei concetti. ${ }^{47}$ In posizione mediana si colloca la redazione della Badia, in quanto si ha una semplificazione dei costrutti sintattici che alleggerisce il testo. Esso si avvicina molto a quello leggibile nel ms. it. 1647 , in cui, però, lo stile, specialmente nei fogli finali contenenti la "giunta", diviene assai dimesso.

Sul piano dei contenuti, si evidenziano delle concordanze ora fra tutti e tre i romanzi, ora esclusive e frequenti di F e B, come pure, occasionalmente, di B e P e, sporadicamente, di F e P. Poiché non c'è un'assoluta identità testuale e tematica tra il Fioravante di Firenze e quello della Badia, è evidente che essi devono essere considerati come due versioni distinte, cosicché, allo stato attuale degli studi, le redazioni italiane della leggenda del cavaliere risultano essere cinque. La scoperta dell'esistenza della stesura del Cod. Conv. Soppr. da Ord. Badia 30 e l'accertamento della sua unicità, insieme al riesame delle relazioni che intercorrono tra le altre compilazioni, determina il superamento dello schema stilato da Mc Arthur, in base al quale da un "primo Libro di Fioravante" sarebbero discese due versioni, una impiegata più tardi ne

46 Barbieri N.I. Un romanzo cavalleresco cit., pp. 34-35.

47 Riguardo all'arcaicità degli aspetti linguistici ed espressivi del Fioravante di Firenze si era già espresso Villoresi M. La letteratura cavalleresca cit., p. 63. 
Il Libro delle Storie di Fioravante e l'altra ritenuta la fonte della stesura settentrionale. ${ }^{48}$ Dalla prima opera sarebbe derivata anche la forma arcaica de Il Romanzo di Francia napoletano. Dalla fusione di elementi ricavabili da quest'ultima, dal Fioravante fiorentino e dalla prima compilazione nel volgare del Nord avrebbero avuto origine, secondo il filologo, anche i primi tre libri dei Reali.

L'impressione generale che si ricava dai dati a nostra disposizione è che ci sia stato, in ogni caso, un "primo Libro di Fioravante", capostipite dell'intera tradizione italiana. Da esso, o da una sua copia posteriore, verosimilmente in volgare toscano, dato che le versioni più numerose, più antiche e più vicine all'archetipo sono state confezionate in questa lingua, potrebbero essere state ricavate due redazioni che si distinguevano, essenzialmente, per la parte riservata all'antenato di Fioravante, chiamato nell'una Fiovo e nell'altra Fiorio. La prima compilazione sarebbe stata poi la fonte per Il Libro delle Storie di Fioravante e anche per una stesura arcaica in lingua napoletana, da cui, in seguito, avrebbe avuto origine la riscrittura tarda de Il Romanzo di Francia. Dalla seconda redazione sarebbero discese sia la Storia di Fioravante sia una primitiva versione settentrionale, la cui copia più recente e palesemente alterata (specie per l'inserimento della "giunta") è rappresentata dal Libro di Fioravante. Più complessa sarebbe stata la genesi de I Reali di Francia, che avrebbero attinto, per i primi tre Libri, sia ad un testo disceso dalla versione napoletana arcaica sia al Fioravante di Firenze sia, infine, alla redazione toscana nata dalla prima scissione interna alla tradizione italiana.

In Fig. 1 si propone uno schema ipotetico che, dal supposto "primo Libro di Fioravante", porta alle cinque compilazioni italiane attualmente conosciute:

48 Per la ricostruzione di Mc Arthur si veda lo schema di Mc Arthur D. Les versions cit., p. 127. In esso, la versione settentrionale da cui discende il Libro di Fioravante del ms. it. 1647 viene definita, con il rischio di un'eccessiva localizzazione linguistica, «version vénitienne». Nelle pagine precedenti (124-126), il critico precisa gli aspetti comuni e distintivi di ciascuna delle quattro redazioni a lui note. 


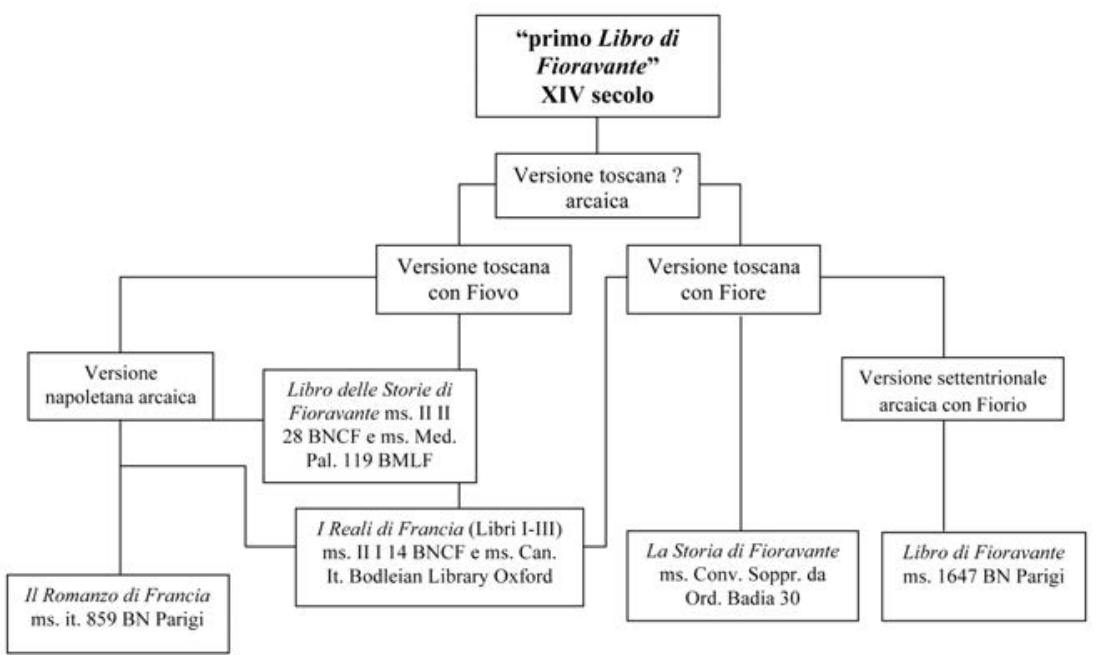

Fig. 1. 